\title{
5G: UNA NUEVA GENERACIÓN PARA UNA NUEVA SOCIEDAD
}

\section{G: A new generation for a new society}

\author{
Robert G. Perea Tamayo ${ }^{1}$ \\ Dirección de Instrumentación Científica de la Agencia Espacial del Perú
}

Recibido: $14-4-2014$

Aceptado: 2-6-2014

\section{Resumen}

El desarrollo de las telecomunicaciones móviles ha mostrado tremendos cambios en los últimos años, pasando de $3 \mathrm{G}$ a 4G, haciendo evidente el crecimiento tecnológico. Es en este momento en el que la investigación se vuelca hacia el desarrollo de la siguiente tecnología. En este artículo se presentan las claves que darán nacimiento a $5 \mathrm{G}$ (quinta generación), exponiendo las investigaciones que vienen realizando diferentes grupos e instituciones a nivel mundial. La visión que presentan es la de un sistema que integra diferentes tecnologías, agrega espectro de frecuencia e incluye a diferentes dispositivos, mostrando que el futuro de la sociedad será influenciado por las telecomunicaciones, tornándose en algo que solo hemos visto como ciencia ficción

Palabras Clave: : inalámbrico, telecomunicaciones, celular, 4G, 5G, IoT.

\section{Abstract}

The development of mobile telecommunications has experienced significant changes in recent years. Moving from the third generation $(3 \mathrm{G})$ to the fourth generation technologies $(4 \mathrm{G})$, makes evident the technological growth. For that reason, research is now concerned with the development of the subsequent technology. In this paper, we introduce the key aspects that give birth to $5 \mathrm{G}$ (fifth generation). We present the research that has being carried out by different groups and institutions around the world. They introduce a system that integrates different technologies, adds frequency spectrum, and includes different devices; proving that the future of society will be influenced by telecommunications, in a way we have only seen in science fiction movies.

Keywords: : wireless, telecommunications, cellular, $4 G, 5 G$, Io T.

1 Máster de Ciencias en Electrónica y Telecomunicaciones, miembro IEEE, Catedrático de la Universidad Alas Peruanas, e Investigador en la Dirección de Instrumentación Científica de la Agencia Espacial del Perú. r_perea@doc.uap.edu.pe, rperea@conida.gob.pe 


\section{INTRODUCCIÓN}

Actualmente los sistemas celulares se encuentran en la cuarta generación o $4 \mathrm{G}$, que define velocidades superiores a 1 gigabit por segundo (Gbps). Basada en LTE (Long Term Evolution), una de las tecnologías de 4G, se viene utilizando OFDMA (Orthogonal Frequency Division Multiplexing Access) para obtener una alta flexibilidad de ancho de banda, ICIC (Inter-Cell Interference Coordination) o coordinación de interferencia entre-celdas para poder utilizar los mismos canales de frecuencia en todas las celdas adyacentes y no adyacentes, Relaying o repetidoras para acceder a los lugares más difíciles, CA (Carrier Agregation) para utilizar más espectro del disponible para una sola comunicaciones, Scheduling o la planificación del recurso tiempo-frecuencia, MIMO (Multiple Input Multiple Output) que consiste en utilizar varias antenas transmisoras y receptoras modificando los patrones de radiación según sea necesario.

A nivel mundial se han desplegado sistemas de 4G basados en LTE o WiMAX (Worldwide Interoperability for Microwave Access) dando servicios de alta velocidad que pueden soportar aplicaciones de video en $\mathrm{HD}$ o videoconferencias.

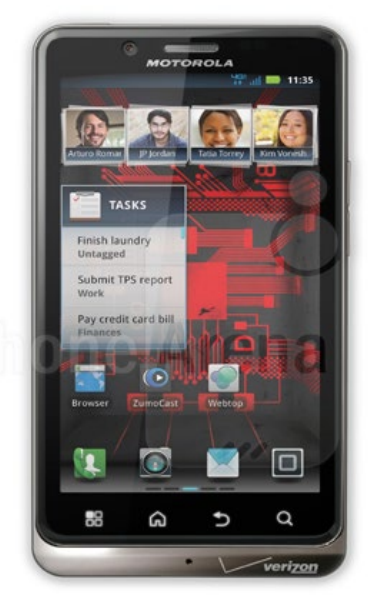

Figura 1. Smartphone 4G.

Fuente: http://mymobileville.com/2011/09/07/at-longlast-the-droid-bionic-arrives-at-verizon-wireless/

Se puede estimar que en pocos años este sistema será bastante usado por usuarios que requieren mayor banda ancha móvil diaria y con servicios de alta calidad. Esto motiva a preguntarse en cuál será el siguiente paso: ¿es posible una mayor velo- cidad? ¿No es suficiente una que permita ver un video HD de forma móvil?

\section{MÉTODO}

Este artículo revisa las publicaciones de instituciones consideradas las más importantes en el área de las telecomunicaciones a nivel mundial, y que tienen como meta el desarrollo de las telecomunicaciones inalámbricas 5G. Se reportan los objetivos de los diferentes proyectos y las visiones con las que se definen los fundamentos de la tecnología $5 \mathrm{G}$ y el futuro de las telecomunicaciones.

\section{GENERACIÓN DE LAS TELECOMUNI- CACIONES INALÁMBRICAS 5G}

Para poder anticipar el desarrollo de la tecnología de las telecomunicaciones es necesario investigar la naturaleza humana, específicamente el cerebro humano.

Se ha comprobado que a medida de que se desarrolla el cerebro, este se vuelve adicto a recibir cada vez mayor información (Lane, 2009). Tal necesidad ha motivado que los diferentes grupos de investigación conciban el término "everything, everywhere and always connected" o "Todo, en todos lugares y siempre conectado", y que refleja el deseo del ser humano para tener siempre y en cualquier lugar cualquier información que quiera. Tal perspectiva fue definida para la informática por M. Weiser (1991), quien en los años 90 vislumbraba una computación que esté en todos lados, embebida en el ambiente y a la vez portátil. A esto se le conoce como "computación ubicua" (Satyanarayanan, 2001), una computación invisible pero que está alrededor de la persona, y que es móvil, es decir se transporta con uno a donde sea.

Los desarrollos en este ámbito se unen a lo que se conoce como inteligencia ambiental (Alcañiz \& Rey, 2005), y que es definida como el siguiente paso de los "smart devices" o dispositivos inteligentes, en donde es el ambiente el que se vuelve "inteligente".

Mirando más en el futuro, el profesor Michio Kaku (2011) define un posible futuro para el año 2100, en diferentes ámbitos, basado en da- 
tos científicos. En el trabajo de Kaku se destaca que para todos los avances tecnológicos desde la medicina, educación, tecnología espacial y hasta la vida cotidiana se requerirá de un sistema de telecomunicaciones que pueda conectar gran cantidad de dispositivos desde cualquier lugar en cualquier momento. Casi todas las visiones futuristas del pasado tienen en común que en la próxima década surgirá una nueva era caracterizada por la total conexión de la vida humana a través de sistemas inalámbricos. Tales predicciones están convirtiéndose en una realidad con el desarrollo de la nueva generación de telecomunicaciones $5 \mathrm{G}$, que no es solo una etapa en la evolución de los sistemas celulares (2G, 3G o 4G) sino que revoluciona los sistemas inalámbricos y las telecomunicaciones globalmente.

La 5G propone integrar diferentes sistemas inalámbricos como Wi-Fi, redes celulares, sistemas de corto alcance con redes de sensores inalámbricos (WSN), comunicaciones de máquina a máquina $(\mathrm{M} 2 \mathrm{M})$ y generando el llamado Internet de Las Cosas (IoT) o interconexión digital de objetos cotidianos con Internet. Es de esperar entonces que, por su propio peso, las diferentes tecnologías se integren en un gran y único servicio centrado en el usuario. Como explica Kaku, citado anteriormente, se puede concebir que en el tema de la salud existan dispositivos monitoreando nuestros signos vitales en busca de alertas tempranas de alguna enfermedad. Para cubrir esta aplicaciónes necesario que exista una red de telecomunicaciones que pueda soportar literalmente miles de dispositivos conectados por cada usuario.

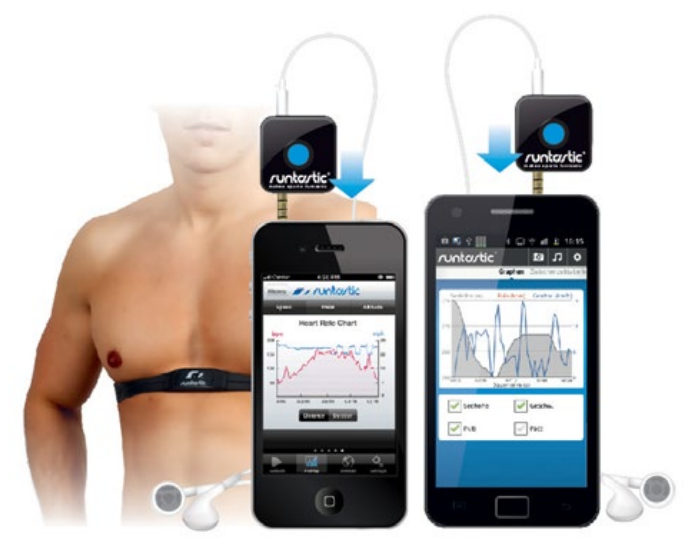

Figura 2. Monitor cardiaco de smartphone. Fuente: http://www.misterrunning.com/runtastic-heart-rate-monitor-for-smartphone-p-5705.html?language =en
Dispositivos como los mencionados existen actualmente y en el futuro cercano, alrededor del año 2020, tendrán una presencia en nuestras vidas tan notoria como la que tienen los smartphones hoy día. Igualmente, términos como ultra-broadband o ultra-banda ancha (NTT DOCOMO, 2014), zero distance conectivity o conectividad a distancia cero (Huawei Europe, 2014) serán las reglas básicas para cualquier servicio de telecomunicaciones.

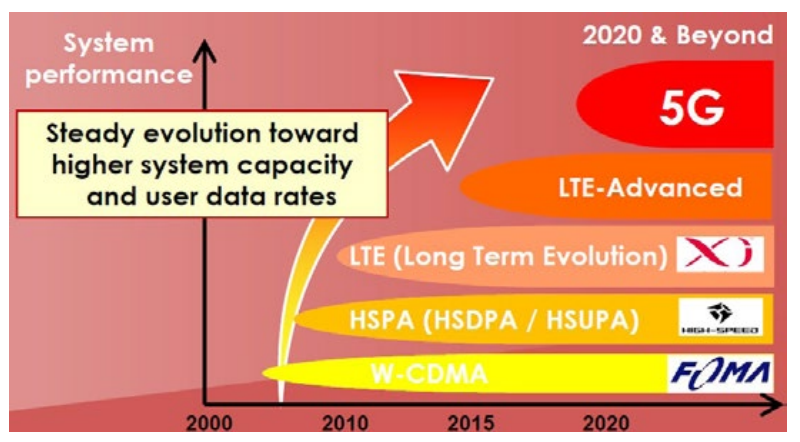

Figura 3. Esquema de la evolución a 5G. Fuente: NTT DOCOMO (2014).

En el último año son varias las entidades que invierten sus recursos para desarrollar la generación 5G. Por ejemplo, el gobierno de Inglaterra en conjunto con empresas líderes en telecomunicaciones creó el Centro de Investigación de 5G (CCSR) con base en la Universidad de Surrey, con una inversión mayor a los $€ 35$ millones. El CCSR busca desarrollar la interfaz aérea para 5G estudiando el espectro de frecuencia, la energía y sistemas satelitales basados en las distintas teorías de telecomunicaciones. Igualmente, trabaja con la Cognitive Radio (CR) o radio cognitiva para tener una interface de radiocomunicaciones inteligente que maneje una gran cantidad de dispositivos conectados de forma eficiente distribuyendo el espectro y administrando los niveles de interferencia. También trabaja en la seguridad y la optimización del sistema de acceso de radio (RAS).

Bajo este entorno aparecen desafíos para el desarrollo tecnológico 5G. Como menciona Fettweis (2012) es evidente que los sistemas de la siguiente generación deben cubrir las necesidades básicas humanas definidas por nuestras constantes de tiempo real, es decir la velocidad con la los sentidos responden a los estímulos externos (tabla 1). 


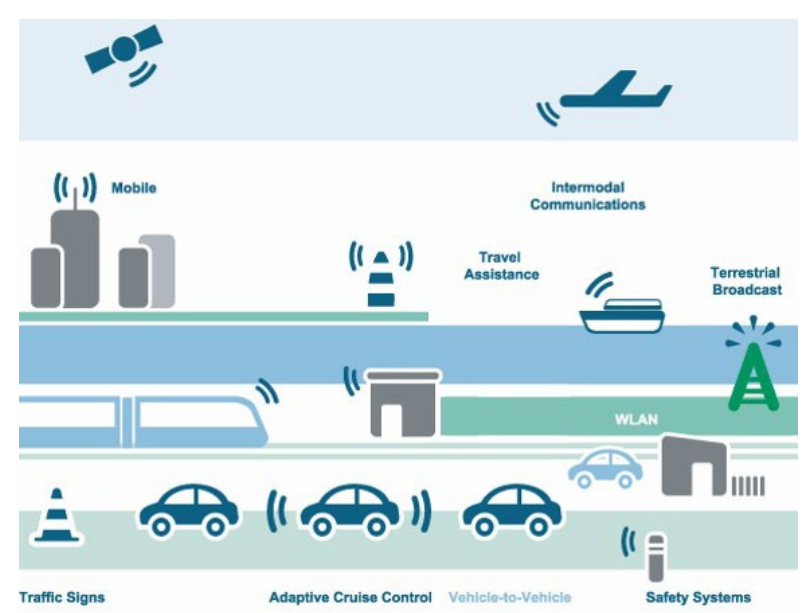

Figura 4. Acceso de Radio. Fuente: CCSR (2013)

\begin{tabular}{|cc|}
\hline Interfaz humana & Tiempo de respuesta \\
\hline Respuesta muscular & $1 \mathrm{~s}$ \\
\hline Oído & $100 \mathrm{~ms}$ \\
\hline Vista & $10 \mathrm{~ms}$ \\
\hline Tacto & $1 \mathrm{~ms}$ \\
\hline
\end{tabular}

Tabla 1. Constantes de tiempo real humano

Se observa que la respuesta muscular al ser la más lenta está actualmente cubierta, al igual que el oído y la vista en en los sistemas de video y audio digital. Pero el tacto con una respuesta menor es el que aún reta las aplicaciones "touch", presentando retardos perceptibles a nuestro sentido. Estos son los niveles de latencia que se deben cubrir para interactuar en "tiempo real" y aun a distancias largas (kilómetros).

Por otro lado, Rappaport (2012), director de NYU Wireless de la Universidad Politécnica de Ingeniería de Nueva York, plantea llenar los espacios libres del espectro electromagnético para abastecer a los sistemas modernos de 5G. Para ello vienen desarrollando la tecnología de ondas milimétricas (millimeter-waves), con la intención de hacer disponible sistemas en frecuencias súper altas, v.g. las bandas de $24 \mathrm{Ghz}, 60 \mathrm{Ghz}, 77 \mathrm{GHz}$, 180Ghz, 330GHz y $380 \mathrm{Ghz}$.

Rappaport (2014) explica que al usarse la banda de $380 \mathrm{GHz}$ para comunicaciones de corto alcance (como sucedería para el IoT) se obtendría un

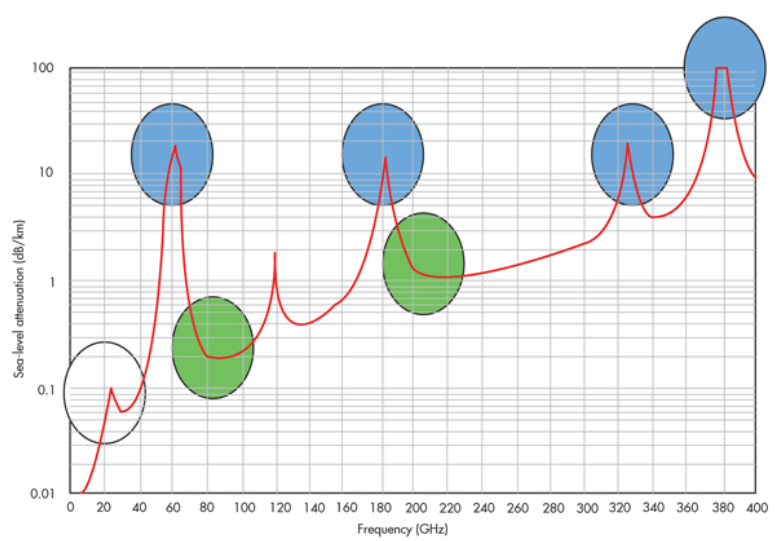

Figura 5. Espectro de ondas milimétricas de Rappaport (2012)

ancho de increíble banda de 10 o $20 \mathrm{GHz}$. Aclara que es importante que las regulaciones gubernamentales se actualicen de modo que estas bandas del espectro, que serán necesarias, sean accesibles

Europa tiene el grupo más grande para el desarrollo de 5G: METIS (Mobile and wireless communications Enablers for the Twenty-twenty Information Society), creado por la Comisión Europea con una inversión €4.2 mil millones de Euros (Mobile World Live, 2014) está conformado por 25 grandes empresas líderes en telecomunicaciones e instituciones académicas (METIS, 2013). Su objetivo es definir los fundamentos para generar un acuerdo europeo sobre los sistemas de telecomunicaciones del futuro que permitan brindar valiosas contribuciones a la pre-estandarización y la regulación de lo que será 5G. Las investigaciones de METIS se centran en en la mejora de eficiencia (costos, recursos y energía), aumento de escalabilidad (alto tráfico y bajo tráfico) y la versatilidad (disponibilidad, movilidad y calidad de servicio) del sistema en conjunto. La multinacional Ericsson, que forma parte de METIS, trabaja bajo la idea de "Networked Society" o Sociedad Conectada (Ericsson, 2013). En su planteamiento, la evolución de los estándares actuales se complementa con las nuevas tecnologías, como aquellas que vengan con comunicaciones M2M y el desarrollo de IoT, que se espera estén disponibles para el año 2020.

Huawei, desde China, se presenta como principal impulsor de la tecnología $5 \mathrm{G}$ planeando invertir más de $€ 444$ millones de euros en los próximos 4 años para su investigación. En su visión define que $5 \mathrm{G}$ deberá tener mil veces la capacidad 


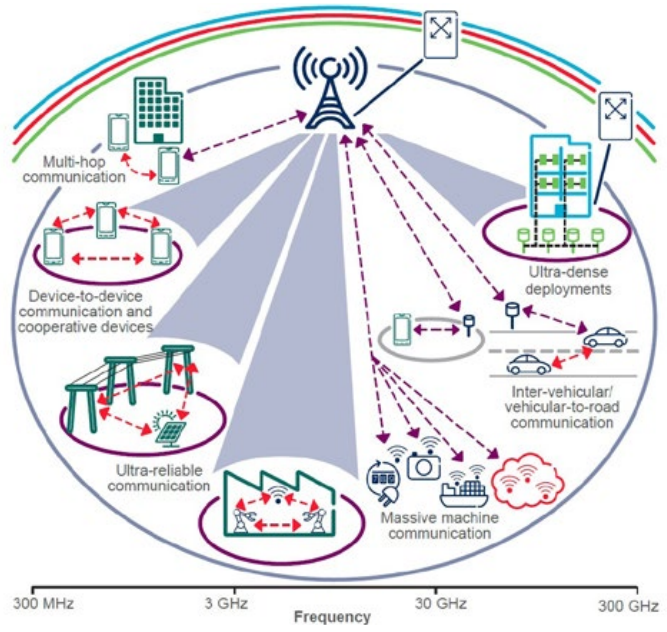

Figura 6. Esquema de las comunicaciones de 5G. Fuente: Ericsson (2013)

actual, 100 mil millones de dispositivos conectados y una velocidad de transferencia de datos superiors a los 10Gbps, con tiempos de respuesta extremadamente cortos. (Huawei Technologies Co., 2013). Pretende desarrollar "Smart Cities" o ciudades inteligentes con gran desarrollo tecnológico. Para definir los requerimientos de los diferentes servicios que aparecerán, Huawei (2013) define el "5G HyperService Cube" o el Cubo de Hiperservicios de $5 \mathrm{G}$ que muestra una cantidad de enlaces, velocidad de datos y latencias relativas a la aplicación involucrada.

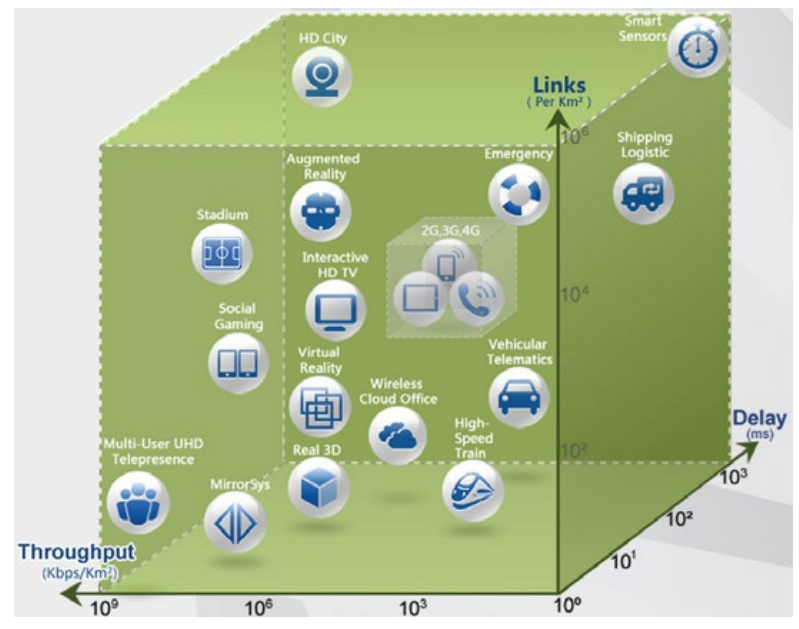

Fig. 8 Cubo de Hiperservicios de 5G.

Fuente: Huawei Technologies Co. (2013)

De acuerdo a las instituciones ya señaladas, la estandarización del 5G debería comenzar en el año 2016 y el producto estaría listo para el ańo 2020.

\section{CONCLUSIONES}

5G utilizará las tecnologías inalámbricas que conocemos hoy en un solo sistema integral proveyendo comunicación masiva desde cualquier dispositivo. Tal comunicación tendrá una latencia sumamente baja, imperceptible para el ser humano, aún en comunicaciones de largas distancias, con una velocidad de transmisión 10 veces mayor a la de $4 \mathrm{G}$, y utilizando el espectro que hasta hace poco era "espacio libre".

Huawei resume $5 \mathrm{G}$ en un solo esquema con múltiples redes, cientos de millones de dispositivos y en un espectro de frecuencia sumamente mayor.

\section{REFERENCIAS BIBLIOGRÁFICAS}

Alcañiz, M., \& Rey, B. (2005). Ambient Intelligence. IOS Press. AliveCor. (2014). AliveCor. Recuperado de http://www.alivecor.com/home

CCSR. (2013). Radio Access System Optimisation . Recuperado el 2014, de http://www.surrey.ac.uk/ccsr/research/ radioaccess/index.htm

Ericsson. (2013). Networked Society Essentials.

Fettweis, G. (2012). A 5G Wireless Communications Vision. Microwave Journal , 55 (12), 24-36.

Huawei Europe. (2014). Huawei’s 5G@Europe Summit 2014. Huawei Technologies Co. (2013). 5G: A Technology Vision.

Kaku, M. (2011). Physics of the Future: How Science Will Shape Human Destiny and Our Daily Lives by the Year 2100. United States: Doubleday.

Lane, C. (Octubre de 2009). The Chemistry of Information Addiction. Scientific American.

METIS. (2013). METIS project overview.

Mobile World Live. (Febrero de 2014). Mobile World Live. Recuperado el marzo de 2014, de http://www.mobileworldlive.com/eu-targets-5g-lead-e4-2b-ri-programme

NTT DOCOMO. (2014). 5G: Next-Generation Mobile Communications System.

Rappaport, T. (Enero de 2014). NYU Wireless' Rappaport envisions a 5G, millimeter-wave future. (T. Parker, Entrevistador) Fierce Wireless Tech.

Rappaport, T. (2012). Wireless Communications in the Massively Broadband Era. Microwave Journal , 55 (12), 46-48.

Satyanarayanan, M. (Agosto de 2001). Pervasive Computing: Vision and Challenges. IEEE Personal Communications.

Weiser, M. (1991). The Computer for the 21st Century. Scientific American 